\title{
Improved proportionate FONLMS algorithm based direct adaptive Turbo equalization for MIMO underwater acoustic communications
}

\author{
Zhuangchun Yang ${ }^{1}$, Tianyi Liang ${ }^{2,3,4, *}$, Zhourong Deng ${ }^{1}$, and Youwen Zhang ${ }^{2,3,4, *}$ \\ ${ }^{1}$ CNOOC Deepwater Development Limited, 518067, China Acoustic Science and Technology Laboratory, the College of Underwater \\ Acoustic Engineering, Harbin Engineering University \\ ${ }^{2}$ Acoustic Science and Technology Laboratory, Harbin Engineering University, Harbin 150001, China \\ ${ }^{3}$ Key Laboratory of Marine Information Acquisition and Security (Harbin Engineering University), Ministry of Industry and Information \\ Technology; Harbin 150001, China \\ ${ }^{4}$ College of Underwater Acoustic Engineering, Harbin Engineering University, Harbin 150001, China
}

\begin{abstract}
In this paper, a novel normalized least mean squares (NLMS) algorithm that jointly updates the efficient of the linear equalizer and soft interference canceller (SIC) in an adaptive turbo equalizer for multiple-input multiple-output (MIMO) underwater acoustic (UWA) communications. To exploit the sparsity of MIMO UWA channels and enhance the convergence speed of adaptive equalization, improved proportionate fast self-optimized NLMS algorithm (IPFONLMS), is proposed to well adapt to sparse channel with the similar complexity as improve proportionate NLMS (IPNLMS) algorithm. Then we extend the proposed algorithm to the adaptive turbo equalization for MIMO UWA communications. The performance of the proposed adaptive algorithm is evaluated by numerical results. Simulation results show that the improved data efficiency and bit error ratio (BER) performance of the proposed receiver is achieved over adaptive turbo equalizer based on the IPNLMS algorithm.
\end{abstract}

\section{Introduction}

In recent years, due to the competition for marine resources, every country has strengthened the research on UWA communications. However, owing to the characteristic of UWA channels, the UWA communications still facing significant challenges[1-3].

UWA communications using MIMO technique shown effective in exploiting the time and spatial diversity characteristics of UWA channel. However, MIMO systems have intersymbol interference (ISI) caused by the multipath phenomenon, which affects communication systems performance[2]. The equalization technique is used to remove ISI. Thus, the turbo equalization (TEQ) techniques for UWA communication is commonly used in large applications[2,3].

In [4], P. Bragard and G. Jourdain demonstrated a linear equalizer that directly adapted its weights via LMS algorithm. However, in the direct adaptation decisionfeedback equalizer (DA-DFE) receiver, the conventional adaptive equalizer algorithms can't obtain a good performance for UWA communications. To improve the performance of UWA communications, a lot of modified LMS algorithms have been investigated[5-7]. In [5], the proportionate normalized least-mean-squares (PNLMS) algorithm is proposed; it converges faster than the normalized NLMS algorithm used in echo cancelers. However, the PNLMS algorithm converges much slower than NLMS algorithm when the impulse response is dispersive. To resolve this problem, a new and simple rule was derived in [7]. In [6], a fast self-optimized LMS (FOLMS) algorithm has been presented. The FOLMS update the step-size with the gradient-search procedure. However, the existing algorithms do not strike a balance between utilize the sparsity of UWA channels and adaptive varying step-size. In order to solve the above issues, in this paper, we present a new adaptive algorithm IPFONLMS to update the coefficients of TEQ for MIMO UWA communications.

The rest of the paper is organized as follows. In section II, the MIMO system model is described and DA-TEQ techniques are briefly described. In section III, the MIMO UWA receiver architecture based on the IPFONLMS algorithm is presented. In section IV presents the numerical results, and in section $\mathrm{V}$, some conclusions are discussed.

Notation: In this paper, we describe matrices and vectors by bold-face italic letters in capital cases and small cases, respectively. The superscripts $(\cdot)^{*},(\cdot)^{T}$ and $(\cdot)^{H}$ represent, respectively, the conjugate, the matrix transpose, and the matrix Hermitian.

\section{System model and architecture}

Corresponding author: angleliangtianyi@,foxmail.com, zhangyouwen@,hrbeu.edu.cn 


\subsection{Transmitter architecture and Receiver architecture}

We consider a $N \times M$ single-carrier MIMO UWA communication system depicted in Fig.1, where $N$ and $M$ is the number of transducers and hydrophones, respectivily. The information bit vector a is encoded by a rate $1 / 2$ recursive systematic convolutional code(RSC) to produce coded bit vector $\mathbf{b}$. The encoded bits in $\mathbf{b}$ are permuted with a random interleaver to produce the interleaved and encoded bit vector $\mathbf{c}$. The bits in $\mathbf{c}$ are grouped and mapped to a finite constellation alphabet $\mathbf{A}$ to produce symbol vector $\mathbf{x} \in \mathbf{A} . \mathbf{x}$ is converted into $N$ parallel transmit streams $\left\{x_{n}(k)\right\}_{n=1}^{N}$ for $N$ transducers by a serial-to-parallel operation.

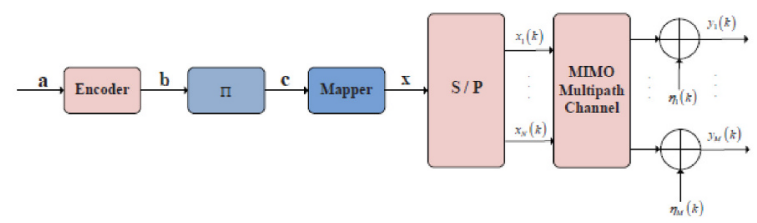

Fig. 1. Block diagram of the MIMO transmitter structure.

At the time $k$, the received signal by $M$ hydrophones can be expressed as

$$
\mathbf{y}(k)=\left(\sum_{l=0}^{L-1} \mathbf{H}_{l}(k) \mathbf{x}(k-l)\right)+\boldsymbol{\eta}(k)
$$

where $\mathbf{x}(k) \triangleq\left[x_{1}(k), \cdots, x_{n}(k), \ldots, x_{N}(k)\right]^{\mathrm{T}}$,

$$
\begin{aligned}
\mathbf{\eta}(k) \triangleq\left[\eta_{1}(k), \cdots, \eta_{m}(k), \cdots, \eta_{M}(k)\right]^{\mathrm{T}}, \text { and } \\
\mathbf{H}_{l}(k) \triangleq\left[\begin{array}{cccc}
h_{(1,1)}^{l}(k) & h_{(1,2)}^{l}(k) & \cdots & h_{(1, N)}^{l}(k) \\
h_{(2,1)}^{l}(k) & h_{(2,2)}^{l}(k) & \cdots & h_{(2, N)}^{l}(k) \\
\vdots & \vdots & \ddots & \vdots \\
h_{(M, 1)}^{l}(k) & h_{(M, 2)}^{l}(k) & \cdots & h_{(M, N)}^{l}(k)
\end{array}\right]
\end{aligned}
$$

where $k$ is time index. $h_{(m, n)}^{l}$ is the $l$-th tap of the length- $L$ equivalent channel between the $n$-th transducer element and the $m$-th hydrophone element. $\eta_{m}$ is the zero-mean valued Gaussian random noise with variance $\sigma_{\eta}^{2}$.

\subsection{DA-TEQ for MIMO systems}

The structure of DA-TEQ is depicted in Fig.2. The DATEQ is made up of feedforward filter, feedback filter and SIC filter. The length of channel is $L=L_{f}+L_{b}+1$, where $L_{f}$ and $L_{b}$ denote the length of precursor and postcursor response, respectively.

We consider an observation window containing $K_{f}+K_{b}+1$ received vectors for DA-TEQ. For convenience, the equalizer output is written as

$$
\hat{x}_{n}(k)=\mathbf{f}_{n}^{\mathrm{H}}(k) \mathbf{r}(k)+\mathbf{b}_{n}^{\mathrm{H}}(k) \breve{\mathbf{x}}_{n}(k)+\mathbf{g}_{n}^{\mathrm{H}}(k) \tilde{\mathbf{s}}_{n}(k)
$$

where

$$
\begin{gathered}
\mathbf{r}(k)=\left[\mathbf{y}^{\mathrm{T}}\left(k+K_{f}\right), \cdots, \mathbf{y}^{\mathrm{T}}\left(k-K_{b}\right)\right]^{\mathrm{T}} \\
\breve{\mathbf{x}}_{n}(k)=\left[\bar{x}_{n}\left(k+K_{f}\right), \cdots, \bar{x}_{n}(k+1), 0, \bar{x}_{n}(k-1), \cdots, \bar{x}_{n}\left(k-K_{b}\right)\right]^{\mathrm{T}} \\
\tilde{\mathbf{s}}_{n}(k)=\left[\overline{\mathbf{s}}_{n}^{\mathrm{T}}\left(k+K_{f}\right), \cdots, \overline{\mathbf{s}}_{n}^{\mathrm{T}}(k), \cdots, \overline{\mathbf{s}}_{n}^{\mathrm{T}}\left(k-K_{b}\right)\right]^{\mathrm{T}}
\end{gathered}
$$

When

$\overline{\mathbf{s}}_{n}\left(k^{\prime}\right)\left\{\begin{array}{l}{\left[\bar{x}_{1}\left(k^{\prime}\right), \bar{x}_{2}\left(k^{\prime}\right), \cdots, \bar{x}_{N}\left(k^{\prime}\right)\right]^{\mathrm{T}},} \\ {\left[\bar{x}_{1}\left(k^{\prime}\right), \cdots, \bar{x}_{n-1}\left(k^{\prime}\right), 0, \bar{x}_{n+1}\left(k^{\prime}\right), \cdots, \bar{x}_{N}\left(k^{\prime}\right)\right]^{\mathrm{T}}, \text { if } k^{\prime}=k}\end{array}\right.$

The $\bar{x}_{n}(k)$ obtained from the priori log likelihood ratio (LLR) of the decoder[2].

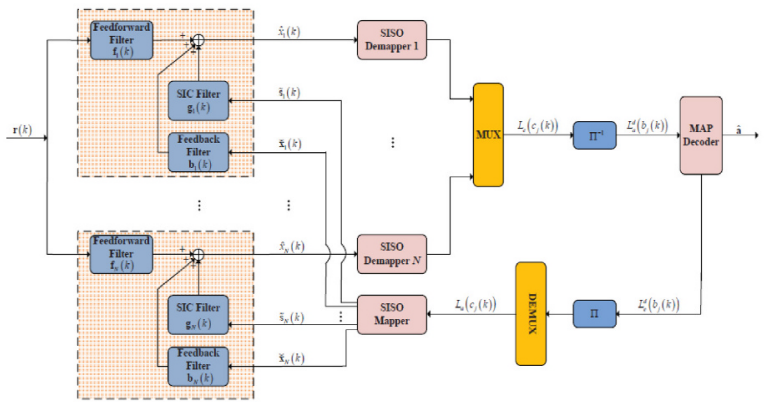

Fig. 2. Block diagram of the DA-TEQ for $N \times M$ MIMO systems.

\section{Proposed IPFONLMS algorithm for MIMO systems}

For the IPFONLMS algorithm, we apply the idea of update step size to the IPNLMS algorithm. Without loss of generality, we take the updating of feedforward filter coefficient for example. The new cost function can be rewritten as

$$
J\left(\mathbf{r}(k), d_{n}(k), \mathbf{f}_{n}(k), \mu\right)=\left|d_{n}(k)-\hat{x}_{n}(k)\right|^{2}
$$

where $d_{n}(k)$ denotes the desired signal. To guarantee the convergence of algorithm, we gave the step size constraint condition. The update equation of step size is expressed as follows

$$
\begin{aligned}
\mu^{f f}(k) & =\mu^{f f}(k-1)-\beta\left(\frac{\partial J}{\partial \mu}\right)(k) \\
& =\left[\mu^{f f}(k-1)-\beta \operatorname{Re}\left(\mathbf{r}(k)\left(e_{n}(k)\right)^{*} \mathbf{G}_{n}^{f f}(k-1)\right)\right]_{\mu_{\min }}^{\mu_{\max }}
\end{aligned}
$$

where

$$
\begin{aligned}
\mathbf{G}_{n}^{f f}(k) & =\frac{\partial \mathbf{f}_{n}(k)}{\partial \mu}=\mathbf{G}_{n}^{f f}(k-1) \\
& +\frac{\mathbf{Q}_{n}^{f f}(k) \mathbf{r}(k)}{\mathbf{r}^{H}(k) \mathbf{Q}_{n}^{f f}(k) \mathbf{r}(k)}\left(e_{n}(k)-\mu^{f f}(k) \mathbf{G}_{n}^{f f}(k-1)\right)
\end{aligned}
$$

and $\beta \ll 1$.

The new update equation can be written as 


$$
\mathbf{f}_{n}(k+1)=\mathbf{f}_{n}(k)+\frac{\mu^{f f}(e(k))^{*} \mathbf{Q}_{n}^{f f}(k) \mathbf{r}(k)}{\mathbf{r}^{H}(k) \mathbf{Q}_{n}^{f f}(k) \mathbf{r}(k)+\delta_{p}}
$$

where $\mathbf{Q}_{n}^{f f}(k)=\operatorname{diag}\left\{q_{n, 0}^{f f}(k), \cdots, q_{n,\left(K_{e q}-1\right)}^{f f}(k)\right\}, \mu$ is the step size, $e_{n}$ is the estimation error of the adaptive filter output with respect to the desired signal and it is $e_{n}(k)=d_{n}(k)-\hat{x}_{n}(k) . \delta_{p}$ is the regularization factor and $\mathbf{Q}_{n}^{f f}$ is a diagonal matrix with the $l$-th diagonal element as follows

$q_{n, l}^{f f}(k)=\frac{1-\alpha}{2 K_{e q}}+(1+\alpha) \frac{\left|\mathbf{f}_{n, l}(k)\right|}{2\left\|\mathbf{f}_{n}(k)\right\|_{1}+\varepsilon}, \quad 0 \leq l \leq K_{e q}-1$

where $\varepsilon$ is a regularization. $|\cdot|$ is the absolute operator and $\|\cdot\|_{1}$ is the $l_{1}$-norm operator. In practice, the selection of $\alpha$ are 0 or -0.5[7]. The updating process of feedback and SIC filters are similar with feedforward. And we can summary the processing of feedback and SIC filters as following

$$
\begin{gathered}
\mathbf{b}_{n}(k+1)=\mathbf{b}_{n}(k)+\frac{\mu^{f b}(k)\left(e_{n}(k)\right)^{*} \mathbf{Q}_{n}^{f b}(k) \breve{\mathbf{x}}_{n}(k)}{\breve{\mathbf{x}}_{n}^{H}(k) \mathbf{Q}_{n}^{f b}(k) \breve{\mathbf{x}}_{n}(k)+\delta_{p}}(11) \\
\mu^{f b}(k)=\left[\mu^{f b}(k-1)-\beta \operatorname{Re}\left(\breve{\mathbf{x}}_{n}(k)\left(e_{n}(k)\right)^{*} \mathbf{G}_{n}^{f b}(k-1)\right)\right]_{\mu_{\min }}^{\mu_{\max }} \\
\mathbf{g}_{n}(k+1)=\mathbf{g}_{n}(k)+\frac{\mu^{s i c}(k)\left(e_{n}(k)\right)^{*} \mathbf{Q}_{n}^{s i c}(k) \tilde{\mathbf{s}}_{n}(k)}{\tilde{\mathbf{s}}_{n}^{H}(k) \mathbf{Q}_{n}^{s i c}(k) \tilde{\mathbf{s}}_{n}(k)+\delta_{p}}(13) \\
\mu^{s i c}(k)=\left[\mu^{s i c}(k-1)-\beta \operatorname{Re}\left(\tilde{\mathbf{s}}_{n}(k)\left(e_{n}(k)\right)^{*} \mathbf{G}_{n}^{s i c}(k-1)\right)\right]_{\mu_{\min }}^{\mu_{\max }}
\end{gathered}
$$

And $\mathbf{G}_{n}(k)$ and $q_{n, l}$ should change the parameters of the corresponding position. Because of the absence of channel information, the DA-TEQ cannot compute the $\mu_{n}^{t}$ and $\delta_{n}^{t}$.The method is detailed description in [2].

\section{Experimental Results}

\subsection{Convergence and Steady-state Analysis of Algorithms}

In this section, we compare the performance of LMStype algorithms in sparse channel. The channel impulse response length is 64. The step size is shown in Fig.3(a), and $\mu(0)$ is set to 0.6 for IPFONLMS algorithm. $\beta=1 \times 10^{-3}$ and $\alpha=-0.5$ for IPFONLMS algorithm. The final NMSE results are averaged over 1000 times under the condition of $S N R=10 \mathrm{~dB}$.

Fig.3(a) shows the performance of three algorithms in time invariant sparse channel. And the channel sparsity level is 10. It can be seen from Fig.3(a) that proposed IPFONLMS algorithm reaches the steady-state faster than other algorithms. The variable step size algorithm will improve performance as the number of iteration increases. We draw a conclusion that the adaptive step size can greatly improve the performance. Under the condition of sudden change sparse channel in Fig.3(b), the proposed algorithm shows better performance than others at sudden change position $n=2500$.

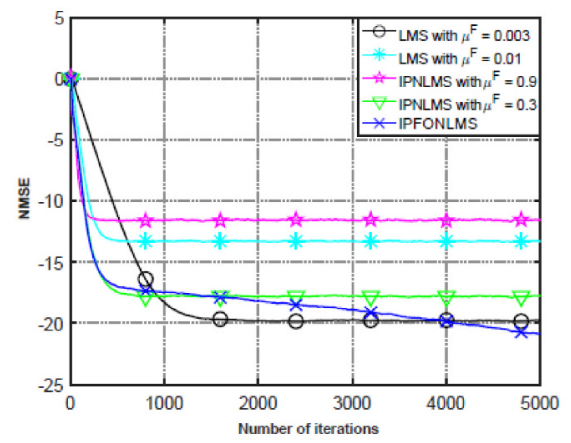

(a) Sparse channel.

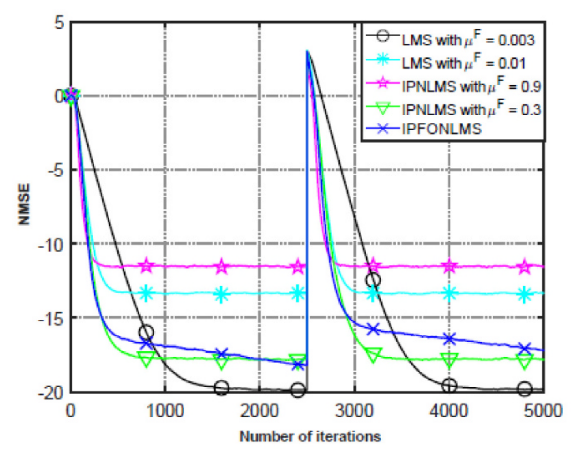

(b) Sudden change sparse channel.

Fig. 3. Learning curves with the channel sparsity level of 10 .

\subsection{Results of System Performance}

For MIMO transmission, the encoding scheme was shown in Fig.1. Two transducers were fixed on the position about 30 metres and 30.25 metres above the surface, and four hydrophones were fixed on the position about 10,10.25, 10.50, and 10.75 metres above the surface. The communication distances were 500 metres. A rate 1/2 RSC channel encoder with generator polynomial $[131,171]_{\text {oct }}$ is used. The data is transmitted in packets. Each packet carries 5000 transmission information, including 500 training sequences in front of the information. The maximum number of data reuse and equalizer iteration were all set to 3 . The initial step size are set to $\mu_{f f}(0)=0.2$ or $0.7, \mu_{f b}(0)=4 \times 10^{-3}$, and $\mu_{\text {sic }}(0)=1 \times 10^{-3}$, respectively. The length of feed forward filter is set to $L$ and the length of feedback filter is set to $2 \times L$. Other relevant parameters are set to $\beta=1 \times 10^{-8}$ and $\alpha=-0.5$. The carrier frequency was $f_{c}=12 \mathrm{kHz}$ and the symbol rate was $4 \mathrm{kps} / \mathrm{s}$. A squareroot raised cosine filter with a roll-off factor of 0.5 , the occupied channel bandwidth of about $12 \mathrm{kHz}$. At the receiver side, the passband width rate was $48 \mathrm{kHz}$. 


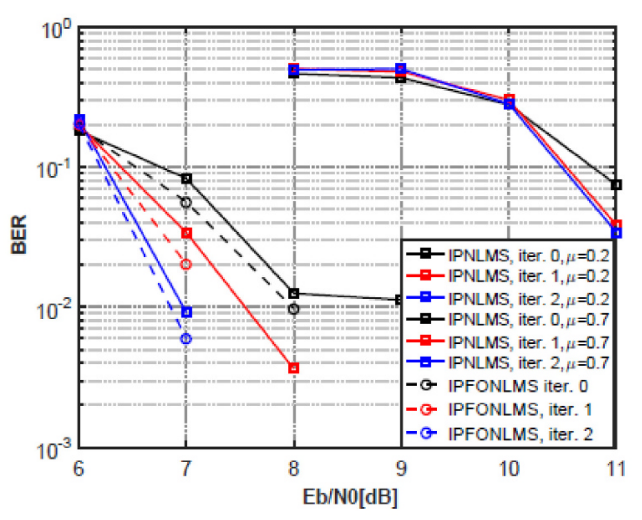

(a)

Fig. 4. Performance of the $2 \times 4$ MIMO DA-TEQ for BPSK.

Fig.4 compares the DA-TEQ performance between IPNLMS algorithm and IPFONLMS algorithm. For $2 \times 4$ MIMO system with BPSK modulation, DA-TEQ leads to a $1 \mathrm{~dB}$ performance penalty at the BER level of $10^{-3}$ at the third iteration. Comparing the different adaptive algorithm, IPFONLMS algorithm based DA-TEQ is better than IPNLMS algorithm based DA-TEQ. At the BER level of $10^{-3}$, the proposed algorithm can get $0.25 \mathrm{~dB}$ gain at the third iteration. In a conclusion, IPFONLMS algorithm manifests better performance than IPNLMS algorithm based DA-TEQ for MIMO UWA communications.

\section{Conclusions}

In this paper, we have proposed IPFONLMS algorithm to update the coefficients of the filters for MIMO UWA communications. By providing variable step-size, the proposed algorithm improves the tracking ability of the adaptive filter in UWA channels. Numerical simulations results show that it can provide a faster convergence rate and a lower MSE than alternative algorithms. And results show that the DA-TEQ based on the proposed algorithm has a superior performance for the MIMO UWA communication system. Therefore, the DA-TEQ sheme based on the IPFONLMS algorithm is a good candidate for practical applications.

The work of Zhuangchun Yang is supported in part by National Science and Technology Major Project under Grant 2016ZX05057002. The work of Y. Zhang was supported in part by the National Natural Science Foundation of China under Grant 61471138 and Grant 50909029, in part by the China Scholarship Council Funding and in part by the Acoustic Science and Technology Laboratory in 2014.

\section{References}

1. M. Stjanovic and J. Preisig, IEEE Communications Magazine, Itextbf $\{47\}, 84-89$ (2009)

2. J. W. Choi, T. J. Riedl, K. Kim, A. C. Singer, and J. C. Preisig, IEEE J. Ocean. Eng. Itextbf $\{36\}$, 473489 (2011)

3. Y. Zhang, Y. V. Zakharov, and J. Li, IEEE Access ltextbf\{6\}, 4955-4973 (2018)
4. P. Bragard and G. Jourdain, Proc. IEEE Int. Conf. Acoustics, Speech, Signal Processing (ICASSP), Itextbf\{3\}, 1425-1428 (1990)

5. D. L. Duttweiler, IEEE Trans Signal Processing, Itextbf $\{8\}, 508-518$ (2000)

6. R. H. Kwong and E. W. Johnston, IEEE Trans Signal Processing, Itextbf $\{40\}, 1633-1642$ (1992)

7. J. Benesty and S. Gay, Acoustic, Speech, and Signal Processing (ICASSP), 2002 IEEE International Conference on, \textbf $\{2\}, 881-1884$ (2002)

8. Y. Zhang, L. Liu, D. Sun, and H. Cui, IET Communications, \textbf $\{9\}, 1867-1876$ (2015) 